\title{
El Departamento de comunicación de las entidades deportivas náuticas
}

Paula González Redondo| paula.gonzalez@protocoloimep.com

Instituto Mediterráneo Estudios de Protocolo

Concepción Campillo Alhama | conchi.campillo@ua.es

Universidad de Alicante

Juan Monserrat Gauchi | juan.monserrat@ua.es

Universidad de Alicante

\begin{tabular}{|c|c|}
\hline $\begin{array}{l}\text { Palabras clave } \\
\text { Comunicación; náutica; }\end{array}$ & Resumen \\
\hline $\begin{array}{l}\text { supervivencia; organización. } \\
\text { Sumario }\end{array}$ & Las entidades deportivas náuticas del siglo XXI se enfrentan a \\
\hline 1. Introducción. & nuevos retos, tales como la relación de los clubes náuticos con la \\
\hline $\begin{array}{l}\text { 2. La comunicación en organizaciones } \\
\text { deportivas náuticas. }\end{array}$ & sociedad actual y su supervivencia, entre otros, que deben afrontar \\
\hline $\begin{array}{l}\text { 3. Método. } \\
\text { 4. Resultados } 41 . \mathrm{El} \text { departamento de }\end{array}$ & con una notable comunicación. La metodología, para conocer la \\
\hline comunicación de las entidades deportivas & gestión de los procesos de comunicación que se realizan en las \\
\hline $\begin{array}{l}\text { náuticas. 4.2. Los recursos humanos del } \\
\text { departamento de comunicación. } 4.3 \text {. Las }\end{array}$ & entidades deportivas náuticas de toda España, se apoya en una \\
\hline $\begin{array}{l}\text { funciones y medios de comunicación del } \\
\text { departamento de comunicación. }\end{array}$ & técnica cuantitativa como es el análisis de contenido. Este análisis \\
\hline 5. Discusión de resultados. & ayuda a entender todo lo referente a las entidades deportivas \\
\hline 7. Bibliografía & náuticas como sistemas de organización. También se emplea la \\
\hline & ca de campo, y la aplicación de la Observación Participar \\
\hline
\end{tabular}

para diseñar un cuestionario que se envía a este tipo de entidades con el objetivo de comparar variables utilizadas en los procesos comunicacionales y obtener así unos resultados que nos llevan a diagnosticar los departamentos de comunicación. Por último los resultados obtenidos confirman que los clubes náuticos les queda mucho camino por recorrer, para implicar a una sociedad del ocio a que siga sus pasos en este siglo XXI. En este sentido, el sector de los clubes deportivos náuticos debe realizar una reflexión sobre la incorporación de los departamentos de comunicación para establecer vínculos con la sociedad.

\section{Cómo citar este texto:}

Paula González Redondo, Concepción Campillo Alhama, Juan Monserrat Gauchi (2018): El Departamento de comunicación de las entidades deportivas náuticas, en Miguel Hernández Communication Journal, nº9 (2), pp. 471 a 490. Universidad Miguel Hernández, UMH (Elche-Alicante). DOI: http://dx.doi.org/10.21134/mhcj.v0i9.258 


\title{
The communication department of nautical sports entities
}

\author{
Paula González Redondo | paula.gonzalez@protocoloimep.com \\ Instituto Mediterráneo Estudios de Protocolo \\ Concepción Campillo Alhama | conchi.campillo@ua.es \\ Universidad de Alicante \\ Juan Monserrat Gauchi | juan.monserrat@ua.es \\ Universidad de Alicante
}

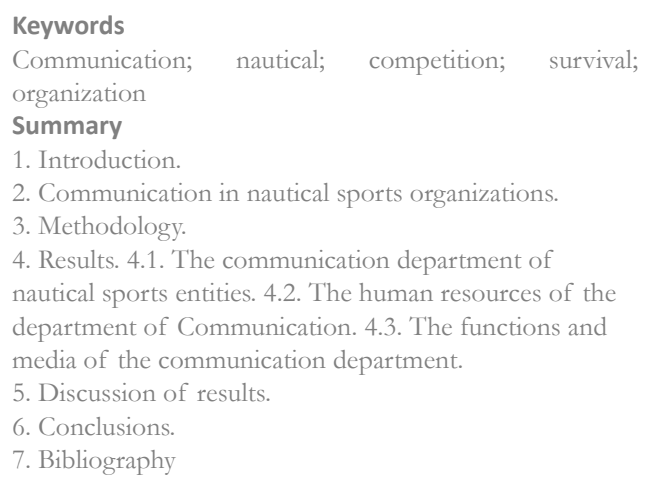

\section{Abstract}

The nautical sports entities of the 21 st century are facing new challenges, such as the relationship of the nautical clubs with the current society and their survival, among others, which they must face with a remarkable communication. The methodology, to know the management of communication processes carried out in nautical sports entities throughout Spain, is based on a quantitative technique such as content analysis. This analysis helps to understand everything related to nautical sports entities as systems of organization. The field technique is also used, and the application of the Participant Observation, to design a questionnaire that is sent to this type of entities in order to compare variables used in communication processes and obtain results that lead us to diagnose the communication departments. Finally, the results confirm that yacht clubs have a long way to go, to involve a leisure society to follow in this 21 st century. In this sense, the sector of nautical sports clubs should reflect on the incorporation of communication departments to establish links with society.

\section{How to cite this text:}

Paula González Redondo, Concepción Campillo Alhama, Juan Monserrat Gauchi (2018): The communication department of nautical sports entities, in Miguel Hernández Communication Journal, nº (2), pp. 471 a 490. Universidad Miguel Hernández, UMH (Elche-Alicante). DOI: http://dx.doi.org/10.21134/mhcj.v0i9.258 


\section{Introducción}

El colectivo de los clubes náuticos, tal como indica Gerardo Pombo García ${ }^{1}$ ha madurado y es por ello que hoy en día estas entidades, asentadas en terreno público, se marcan como objetivo el definirse como clubes deportivos del siglo XXI, con todo lo que ello comporta: su relación con las instituciones, su gestión y su papel en la sociedad. La Confederación Española de Asociaciones de Clubes Náuticos (CEACNA), durante la celebración del I Simposio Nacional de Clubes Náuticos (2011) se celebró con el fin de aunar los esfuerzos que los nuevos retos del siglo XXI presentan a este tipo de entidades náutico deportivas. Cuestiones, tales como: la relación de los clubes náuticos con la sociedad actual, los clubes deportivos del siglo XXI y retos del futuro, entre otros, fueron los temas que se plantearon para garantizar la supervivencia de este tipo de entidades ${ }^{2}$.

Por lo tanto, las entidades náuticas deportivas en este siglo XXI se encuentran con una mayor competencia y con una sociedad del ocio que implica que el sector de los clubes náuticos se marque una reflexión sobre su papel en la sociedad actual ${ }^{3}$. Una cuestión que llega en un momento esencial para la evolución del papel tradicional de los clubes náuticos en España porque el usuario, mediante las nuevas tecnologías de la información, se ha hecho con el poder de la comunicación a golpe de un solo click. De este modo, entre los retos más importantes para estas entidades figuran, por una parte, comunicar que la náutica está al alcance de todos, por otra, intentar anular el estigma de que navegar es sólo para clases privilegiadas. Estos son los dos grandes desafíos para la supervivencia de todos los clubes náuticos cuyas organizaciones, en algunos casos, son centenarias.

Tal como nos hemos referido anteriormente, este tipo de entidades deben definir una estrategia de posicionamiento e imagen de la náutica deportiva en España para comunicar que la náutica está al alcance de todos y disipar la creencia actual de que es tan sólo para clases privilegiadas. Una labor primordial para situar a estas entidades náuticas deportivas en igualdad de condiciones que otros sectores de ocio para la sociedad. Así mismo, transmitiendo esta imagen se facilitaría el conocimiento de la náutica deportiva por el gran público, que es el potencial consumidor de la oferta náutica y deportiva ofrecida por cada uno de los clubes náuticos.

En este sentido, la Federación de Vela de la Comunidad Valenciana (FVCV) asegura que se observa como estas entidades deportivas apuestan por la comunicación y por las nuevas tecnologías para transmitir su actividad social a toda la sociedad. Una comunicación que resulte comprensible y eficaz utilizando todos los medios a su disposición, y donde internet y las nuevas tecnologías van a desempeñar un papel fundamental. Las estrategias de comunicación aplicadas a la organización de los clubes náuticos es un tema, que cada vez más, está presente en este tipo de entidades que quieren transmitir todo lo concerniente a la náutica a través de los medios de comunicación.

${ }^{1}$ El Presidente de la Real Federación Española de Vela (2001-2012) lo menciona en el I Simposio Nacional de Clubes Náuticos celebrado el 18 de octubre de 2011 en el Salón Náutico de Barcelona. Disponible en: http:/ /www.fvrm.es/index.php?option=com_content\&view $=$ article\&id $=231 \% 3 \mathrm{As}$ imposio-nacional-de-clubes-nauticos-2011\&Itemid=1. [Consulta: 5 mayo 2012]

${ }^{2}$ Disponible en: http://www.ceacna.com/noticias2011_1.html. [Consulta: 1 junio 2012]

${ }^{3}$ Organizado por la Real Federación Española de Vela (RFEV) con la colaboración de la Confederación Española de Asociaciones de Clubes Náuticos (CEACNA) y la Asociación Española de Clubes Náuticos (AECN). Disponible en: http://www.acncv.org/wp-content/ uploads/2014/07/CONCLUSIONES-SIMPOSIO-NACIONAL-DE-CLUBES-20111.pdf. [Consulta: 2 septiembre 2014] 
Así es como se reflejó en el II Simposio de Clubes Náuticos ${ }^{4}$ celebrado en Palma de Mallorca, 28 y 29 de noviembre de 2015, en el que los representantes de los distintos clubes náuticos que asistieron concluyeron ${ }^{5}$ que el marketing online es una herramienta adecuada de relación porque ayuda a compartir experiencias y sensaciones que se quieren transmitir a los socios, clientes y consumidores de servicios. De este modo, este tipo de entidades deben saber con claridad qué imagen quieren transmitir para centrarse en los valores y emociones que aportan sus servicios.

Los clubes náuticos tratan de concienciar a sus públicos de que su principal razón de ser es fomentar el deporte náutico. De este modo, estas entidades no pueden ser concebidas como simples parkings de barcos porque pierden su valor diferencial y, con él, el argumento de su permanencia.

De este modo es como se planteó en el III Simposio de Clubes Náuticos ${ }^{6}$ celebrado en Alicante, 6 y 7 de octubre de 2017, en el que se expuso la necesidad de potenciar y apostar de manera decidida por la profesionalización de los departamentos de comunicación de las entidades deportivas náuticas. Por lo tanto las nuevas tecnologías de la comunicación son una ventana, al alcance de cualquier público, para acceder a la oferta náutica de estas entidades sin ánimo de lucro.

Estas nuevas tecnologías, tal como asegura Cole (2016:15), están actuando como plataformas difusoras de información, noticias y valoraciones, que son de gran interés para el conjunto de organizaciones deportivas. Lobillo y Muñoz (2016:195-214) inciden en que las redes sociales permiten elaborar y compartir contenidos generados tanto por las organizaciones como por el resto de usuarios. Por tanto, estas herramientas son de gran interés para las entidades deportivas náuticas porque permiten interaccionar, de manera directa, con sus diversos públicos.

Es por ello que en el sector deportivo las redes sociales son reconocidas como una importante herramienta de marketing, ya que puede obtener beneficios a la hora de vender un servicio o actividad deportivo (Olabe, 2015:83-104). Así también lo considera Rojas y Panal (2017:1-19) que aseguran que las red social Instagram tiene unas funcionalidades propias que facilitan la interacción entre sus usuarios, algunas parecidas a otras redes sociales, y que son de gran utilidad para la comunicación.

Esta investigación se centra en los procesos de comunicación y gestión relacional de las entidades deportivas náuticas para fomentar la actividad deportiva. Así mismo, los clubes náuticos son el primer eslabón de la cadena de acceso a la mar de miles de ciudadanos, nacionales y turistas. Por lo tanto, las principales metas que día tras día quieren alcanzar estas entidades son acercar a la sociedad la labor deportiva y económica que desarrolla cada club náutico, seguir trabajando por un modelo social que acerque la náutica a todas las economías, y trabajar unidos para comunicar el valor social de nuestras entidades y la influencia de nuestra masa crítica como grupo.

Partimos de la hipótesis de que la mayoría de las entidades náuticas no le otorgan suficiente importancia a la comunicación que dirigen hacia su público (H.1). La $2^{\mathrm{a}}$ hipótesis se plantea en torno a que la comunicación, que realizan este tipo de sociedades sin ánimo de lucro la desempeña personal especializado que cumple funciones en otros departamentos (H.2). En cuanto a la $3^{\text {a }}$ hipótesis las entidades deportivas náuticas siguen una comunicación convencional, aunque se tiende hacia las nuevas tecnologías para difundir sus actividades a los socios (H.3).

${ }^{4}$ Disponible en: http://www.simposioclubsnauticos.es [Consulta: 15 febrero 2016]

${ }^{5}$ Disponible en: http://www.simposioclubsnauticos.es/uploaded_files/

Document_2190_20151214181055_es.pdf [Consulta: 15 febrero 2016]

${ }^{6}$ Disponible en: http:/ /www.simposioclubsnauticos.es [Consulta: 10 noviembre 2017] 
Su verificación requiere de la consecución de los siguientes objetivos:

O.1. Conocer si existe un departamento que gestione la comunicación dentro de la entidad. O.2. Analizar los recursos humanos que desempeñan las funciones del departamento de comunicación de las entidades.

O.3. Estudiar qué funciones y medios emplean los departamentos de comunicación de los clubes náuticos para comunicarse con sus socios.

Todos ellos nos permitirán conocer más de cerca cómo son los departamentos de comunicación del sector deportivo náutico.

\section{La comunicación en organizaciones deportivas náuticas}

Al igual que cualquier otra organización, según Billorou (1997:279), las entidades deportivas sin ánimo de lucro, cuando realizan sus campañas publicitarias, siguen una serie de etapas tales como:

1) Etapa de planificación: que es la etapa en la que se asientan las bases acerca de todas las actividades que se van a desarrollar a lo largo del año. Teniendo en cuenta qué actividad se va a anunciar, el presupuesto de esa campaña, los medios que se van a utilizar y su planificación por medios y soportes, contenido del mensaje y a quién va a ir dirigida la campaña.

2) Etapa de decisión: en la que se presenta la campaña a la dirección y éste la aprobará, la rechazará o solicitará modificaciones al material presentado. Una vez aprobada la campaña se procede a su ejecución.

3) Etapa de desarrollo y exposición: como etapa de ejecución de la planificación especificada por la organización, tras su supervisión y aprobación. Es la etapa en la que el anuncio se expone en los medios contratados.

4) Análisis de los resultados: los resultados deben medirse conforme a los objetivos, de forma que habrá que acudir a éstos y compararlos con el resultado final. La eficacia puede medirse tanto en términos cuantitativos como cualitativos.

Báez (2000:226) añade que toda campaña de comunicación consta del establecimiento de unos objetivos, la recopilación de informaciones, la determinación del "eje" de la campaña, la creación de la propuesta, la elaboración del mensaje, el plan de medios y soportes, presupuesto, control y evaluación. De este modo, no podemos olvidar que en este tipo de entidades deportivas, al igual que cualquier otra organización, podemos encontrarnos con departamentos de comunicación poco complejos en cuanto a su estructura y en el que el responsable se encarga de realizar todas las funciones de comunicación y gestión relacional que se le asignen, o bien con un departamento de comunicación de estructura compleja, consolidado y con experiencia, en el que el responsable y su equipo de trabajo asumen funciones mucho más específicas y especializadas.

Xifra (2011) se refiere a cuatro fases, tales como investigación, planificación, ejecución (comunicación) y evaluación, como el proceso lógico que ayuda a una organización a alcanzar la meta de crear, mejorar y/o mantener relaciones beneficiosas con sus públicos. Afirma que la acción de gestión relacional (que se identifica con la función de relaciones públicas en las organizaciones) es la actividad desarrollada desde una empresa u organización de crear, mantener o mejorar la confianza del público al que se dirige la empresa o institución a través de una comunicación bidireccional. 
Por lo tanto las organizaciones deportivas apuestan por las campañas de comunicación para trasladar sus mensajes a los diferentes medios y éstos se hagan eco del contenido de los mismos, y así crear expectativas de compra, sensibilizar a la opinión pública, aportar datos informativos y contenidos comerciales (Comunicación global, 2015).

Si nos centramos en las funciones de los departamentos de comunicación de este tipo de entidades, observamos que no hay una gran diferencia con otras organizaciones de diversa índole. Billorou (1997:280) las clasifica de la siguiente forma:

- Funciones de dirección: para analizar las necesidades de comunicación, preparar el plan de comunicación, determinar el presupuesto, planificar las campañas publicitarias, determinar las políticas de comunicación, analizar a la competencia y en caso de necesitar una agencia de publicidad, seleccionar una de ellas.

- Funciones de coordinación: a nivel externo, suministrando información a la agencia o consultora externa; evaluar, aprobar o rechazar las soluciones que ésta propone; supervisar las tareas; evaluar el proyecto comunicativo y de gestión relacional a través de los resultados. A nivel interno, cooperando con cualquier área de la empresa, coordinando las investigaciones que sustentan las estrategias comunicativas organizacionales.

- Funciones de producción: con el desarrollo de todo el material publicitario, de promoción o packaging, entre otros; es fundamental la gestión y negociación con proveedores externos.

- Funciones administrativas: en las que se controla que todo lo contratado en los medios realizando su seguimiento; es decir, controlar la inversión que se ha hecho a partir del presupuesto aprobado.

De Moragas (2007) afirma que, desde mediados del siglo XIX y hasta finales del XX, la comunicación se generaba en las propias organizaciones y era el evento en sí el elemento emisor de los mensajes que recogían los medios de comunicación, a través de acciones de publicity, para distribuirlos a las audiencias de esos deportes. Será a partir del siglo XXI con la aparición de las nuevas tecnologías de la comunicación e información (TIC) cuando los procesos de los flujos informativos cambian. $\mathrm{Y}$ añade que, aunque se mantienen esas constantes, en esta época la comunicación es bidireccional, ya que han aparecido nuevos soportes comunicativos vinculados a las tecnologías de la comunicación e información (TCI) que permiten a las audiencias participar activamente en ese proceso bidireccional como emisores y no sólo como receptores.

Es por ello que en esta nueva era las redes sociales juegan un papel importante siendo los nuevos sistemas de comunicación, que se hacen hueco en los departamentos de comunicación de las organizaciones. Los responsables de comunicación de este siglo tienen que participar de manera activa en sus redes, tales como Twitter, Linkedin y Facebook, para ejercer su influencia ${ }^{7}$. Por lo tanto las actividades diarias que más se divulgan en las redes sociales, tales como competiciones, ruedas de prensa, entrevistas, entre otras, son el elemento primordial de los seguidores y con mayor repercusión en los medios (Castillo, Fernández y Castillero, 2016: 239-254).

De este modo, Llopis-Goig (2016:86-89) considera que la principal fuerza impulsora del deporte comienza a partir de los procesos de globalización y mediatización en la sociedad digital. Por lo tanto, en el mundo del deporte la revolución digital ha producido una proliferación de las retransmisiones compartidas desde la plataforma de Internet (David y Millward, 2015). En este sentido Llopis-Goig (2016:86-89) asegura que la revolución digital supone un desafío a los

${ }^{7}$ Disponible en: http://www.dircom.org/images/Anuario-Dircom-2015.pdf [Consulta: 20 junio 2018] 
canales de distribución, creando la posibilidad de compartir libremente transmisiones deportivas en vivo a través de plataformas de Internet; es decir, las nuevas aplicaciones de Internet y dispositivos móviles no han sustituido a la televisión como principal pantalla para el consumo deportivo.

El comienzo del marketing deportivo en este tipo de organizaciones deportivas sin ánimo de lucro, tal como afirma Sanahuja (2013:126-143) se produce a mediados del S. XX. Los grandes clubes deportivos desarrollan actuaciones muy complejas que contrastan con los clubes más modestos, que se afanan en consolidar sus áreas funcionales de comunicación y marketing asignando recursos humanos de forma permanente. En este sentido Paché e Ika (2017:30-34) consideran que, a día de hoy, el marketing y las relaciones públicas prestan una especial atención a todos los elementos que influyen en la comunicación corporativa de los equipos deportivos.

Sanahuja (2013:126-143) incide en el hecho de que en ese contexto se producen una serie de disfunciones, tales como la dirección paternalista en bastantes de estas entidades más modestas, propiciadas por estructuras familiares y una total ausencia de investigación sobre el sector y el entorno propio, la toma de decisiones irracionales, la singularidad de un mercado en el que no se identifican con estudios serios y rigurosos sus necesidades, amenazas, riesgos, dificultades y fortalezas de los productos o servicios a ofrecer, la complejidad de sus actores, la presión mediática, el divismo de los presidentes, así como un modelo de negocio insostenible con disparidad de dimensiones, basado en una distribución no equitativa de los ingresos por derechos de retransmisión y dependencia sobre éstos.

Con el paso del tiempo, y especialmente a partir de la década de los 90, se fue tendiendo a la profesionalización del área o estructura de comunicación, tal como asegura Olabe (2009:121137); lo que supuso, a partir del siglo XXI, según afirma García (2005:191-194), un crecimiento de dichas estructuras del 50\% respecto al 3\% durante el siglo XX.

El principal objetivo de comunicación de las entidades deportivas sin ánimo de lucro, según Olabe (2009:121-137), fue mantener relaciones comunicativas con los mass media, por lo tanto no es de extrañar que este tipo de organizaciones deportivas hayan apostado por integrar en sus estructuras empresariales un departamento de comunicación que realiza sobre todo funciones de relación con los medios de comunicación, aunque en muchas ocasiones desempeñan también otras labores complementarias y que ya hemos identificado cuando hemos hablado de la comunicación estratégica en las organizaciones. Estas entidades al igual que la mayoría de las organizaciones e instituciones deportivas, basan sus estrategias comunicativas en la relación con sus diversos públicos objetivos aplicando herramientas y acciones propias de la comunicación off the line que se integran en procesos below the line ${ }^{8}$.

De este modo, la implementación de las nuevas tecnologías de la comunicación y la información (TIC) ha supuesto que muchas de estas organizaciones deportivas sin ánimo de lucro se hayan sumado a la tendencia actual de crear su propio portal corporativo (más como herramienta de prestigio que con un uso estratégico), dejando de lado las múltiples posibilidades que ofrece el nuevo medio, tal como los blogs, e-boletines, microsites o la utilización de redes sociales, entre otros. Olabe (2015:83-104) asegura que en los últimos años se ha incrementado el control de información a través de sus propios soportes comunicativos, Internet, redes sociales, sitio web corporativo y componentes digitales. De este modo, los gabinetes de prensa se han convertido en los "gatekeepers", en cuanto a que antes facilitaban la información y ahora son los que la

${ }^{8}$ Las mismas acciones de comunicación que realizan en los medios de comunicación convencionales: televisión, radio, prensa y exterior, las integran en los medios de comunicación alternativos: anuncios online como por ejemplo en foros o blogs, carteles o por medio de displays, en el transporte público, letreros luminosos, en series de televisión, etc. 
controlan y gestionan, y la hacen periodística.

Por lo tanto las herramientas 2.0, tal como indica Paloma Cabrera López ${ }^{9}$ han hecho que las organizaciones se reinventen mediante una comunicación más fluida y cercana. Los nuevos canales permiten a las entidades escuchar y prestar más atención a sus diferentes públicos. Es por ello que la comunicación online ha modificado la forma de actuar de las entidades ante la sociedad.

Ante este panorama, los departamentos de comunicación de las entidades deportivas no han comprendido todavía el enorme potencial comunicativo que suponen estas herramientas desde una doble perspectiva:

$\checkmark$ Potenciar el mix de comunicación de la organización, gracias al complemento que supone la comunicación on-line con la comunicación off-line.

$\checkmark$ La posibilidad de posicionar la imagen corporativa, tanto en los públicos de la organización como en públicos potenciales. Como consecuencia, Olabe (2009:121137) asegura que estas organizaciones desaprovechan la oportunidad de reforzar su reputación y su imagen corporativa.

A día de hoy los gabinetes de comunicación se encargan de gestionar la comunicación del club deportivo con los medios de comunicación, crear noticias positivas sobre su entidad y estar atentos a aquellos periodistas críticos con la gestión del club. De este modo la imagen de estas entidades sin ánimo de lucro es tan importante que el marketing, la dirección de comunicación y la comunicación son términos, que ya forman parte del organigrama de estas instituciones deportivas (Olabe, 2015:83-104).

De Asís, Gross, Lillo y Caro (2002:17) consideran, en este sentido, que los medios usados por cualquier entidad deportiva sin ánimo de lucro son muy variados y su número tiende a ampliarse a medida que ésta crece y madura su política de comunicación. Y así, Olabe (2009:121-137) y Herranz (2010:147-148) aseguran que estas entidades necesitan, como cualquier otra organización, empresa o institución, trasladar su imagen a la sociedad para conseguir sus objetivos, por lo que deben recurrir a la comunicación. En la propia dinámica de la sociedad actual predomina la información sobre diversos servicios, y en el caso de la cultura organizativa se atiende a la identidad, calidad y motivación de los grupos de interés. Por lo tanto, las organizaciones deportivas sin ánimo de lucro deben apostar por la comunicación estratégica.

Olabe (2009:121-137) y García (2005:191-194) afirman que el director de comunicación de estas organizaciones deportivas sin ánimo de lucro suele ser miembro incluso de la propia estructura directiva. Ambos autores manifiestan que el Dircom, como responsable máximo del diseño de las estrategias comunicativas-relacionales y su posterior ejecución a través de diversas acciones, debe asumir sus responsabilidades desde la orientación de la planificación comunicativa y relacional, vinculada a su mapa de públicos, con estrategias y acciones reflexionadas y ejecutadas en función de sus objetivos comunicativos.

Tal y como asegura Vázquez (2004:9-17), el director de comunicación de este tipo de organizaciones debe desempeñar tres funciones básicas en su tarea profesional:

1) La función periodística: vinculada a la transparencia, en sus acciones y en la

${ }^{9}$ La Directora de Marketing y Comunicación de Accenture para España, Portugal, África e Israel lo menciona en el Anuario de la Comunicación 2015. Disponible en: http://www.dircom.org/images/ Anuario-Dircom-2015.pdf [Consulta: 20 junio 2018] 
comunicación de la organización a sus públicos.

2) La función de Relaciones Públicas: encarnada en la figura de su actuación como portavoz de la organización a la que representa.

3) Las funciones de marketing y publicidad: como coordinador de este departamento específico que aparece en muchas ocasiones desligado del propio departamento de prensa.

Entre las funciones que este autor asigna al Dircom en las organizaciones deportivas, además de la comunicación interna y externa, también se encuentran otras que trascienden el marco puramente informativo, tales como: la comunicación institucional, la portavocía de la organización, la creación de imagen corporativa, la comunicación de crisis, implantar y desarrollar la cultura corporativa, el desarrollo de la política social e institucional, las publicaciones y audiovisuales de la organización, la publicidad en coordinación con el departamento de marketing, el patrocinio y mecenazgo, el asesoramiento a la presidencia y al consejo directivo, la relación pública e institucional, la realización de todo tipo de actos, eventos y el protocolo, las relaciones con los medios de comunicación y la e-comunicación.

Consideramos que la comunicación de las organizaciones deportivas sin ánimo de lucro está integrada por diferentes facetas comunicativas como ocupación profesional, y dicho rol viene condicionado por la estructura, naturaleza y características de la propia entidad, por sus necesidades comunicativas y relacionales, entre los que se incluyen los vinculados con la comunicación. Estos departamentos realizan acciones comunicativas para públicos externos e internos, con especial énfasis en la gestión de las relaciones con los medios de comunicación.

En este sentido, los gabinetes de comunicación en este tipo de organizaciones buscan condicionar la agenda de los medios de comunicación, sus rutinas y contenidos, con el uso de técnicas y herramientas comunicativas adaptadas a sus necesidades, tales como las ruedas de prensa, los comunicados, la concertación de entrevistas y reportajes o el uso de la web corporativa con la actualización diaria de contenidos.

A partir de estas conclusiones se plantean las hipótesis de nuestra investigación sobre la comunicación de estas entidades deportivas náuticas y el tipo de comunicación que desempeñan este tipo de sociedades sin ánimo de lucro.

\section{Método}

El siguiente estudio tiene como objetivo conocer la gestión de los departamentos de comunicación de las entidades deportivas náuticas. Para ello se ha seguido una metodología de análisis de contenido, a través de fuentes documentales, sobre entidades deportivas náuticas como sistemas de organización de este tipo de asociaciones. Seguidamente y mediante la técnica de campo, y la aplicación de la Observación Participante, observaremos y recogeremos toda la información posible para diseñar un cuestionario, que nos ayudará a recabar información de la población seleccionada para el estudio de investigación. Esta encuesta compuesta por 33 preguntas, en su mayoría cerradas cuyas respuestas ya están acotadas y fáciles de responder, se ha diseñado en base a los resultados obtenidos mediante la Observación Participante en el Real Club de Regatas de Alicante. Este cuestionario ha sido enviado por correo electrónico, entre el 15 de julio y el 31 de agosto de 2015, a 164 entidades deportivas náuticas, que forman parte de la Real Federación Española de Vela (RFEV), con el objetivo de comparar las variables utilizadas 
y obtener así los resultados. Las 164 enseñas representan nuestra población objetivo, habiendo obtenido respuesta de 79 de ellas. Este porcentaje de respuestas sobre el universo de estudio es de un $48,17 \%$.

\section{Resultados}

A continuación, se exponen los resultados obtenidos en relación a los objetivos planteados con el fin de analizar las características fundamentales de los departamentos de comunicación en este tipo de entidades.

\subsection{El departamento de comunicación de las entidades deportivas náuticas \\ En relación con el O.1. en el siguiente gráfico se muestra la existencia del departamento de comunicación dentro de la entidad.}

Gráfico 1. Existencia del Dpto. de Comunicación en las entidades deportivas náuticas

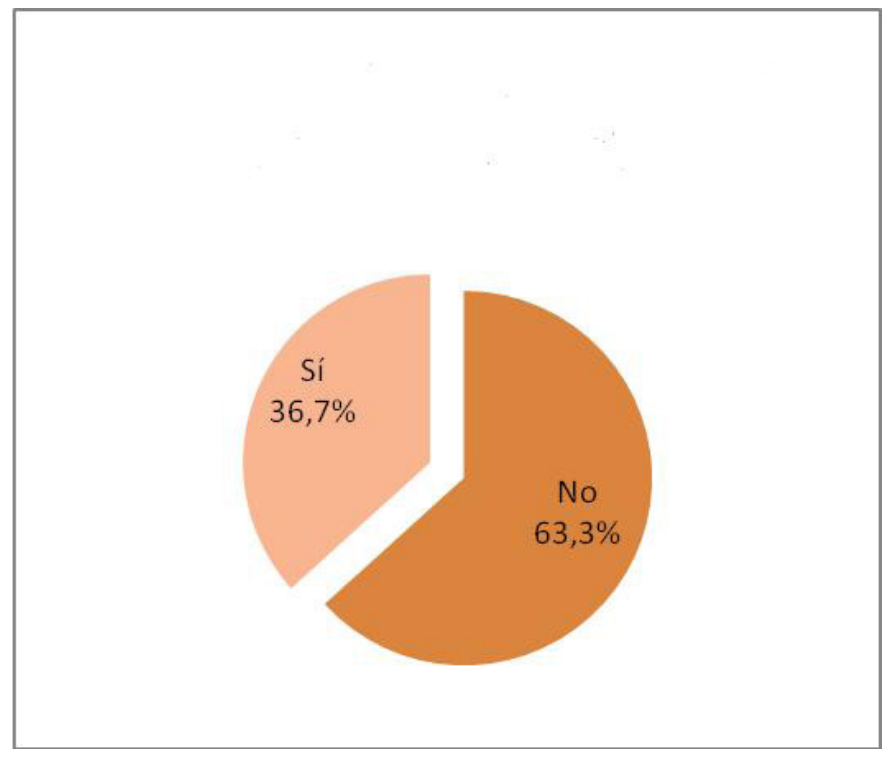

Fuente: Elaboración propia

Los resultados evidencian que el 63,3\% de estas entidades no tienen departamento de comunicación. En cambio el 36,7\% sí apuestan por la existencia de un departamento que gestione la comunicación dentro de la entidad. De este modo, los datos son muy reseñables puesto que la mayoría de entidades deportivas náuticas no valoran o consideran que ese departamento esté presente en su entidad.

\subsection{Los recursos humanos del departamento de comunicación}

Gráfico 2. Gestión y ejecución de la función de comunicación 
Fuente: Elaboración propia

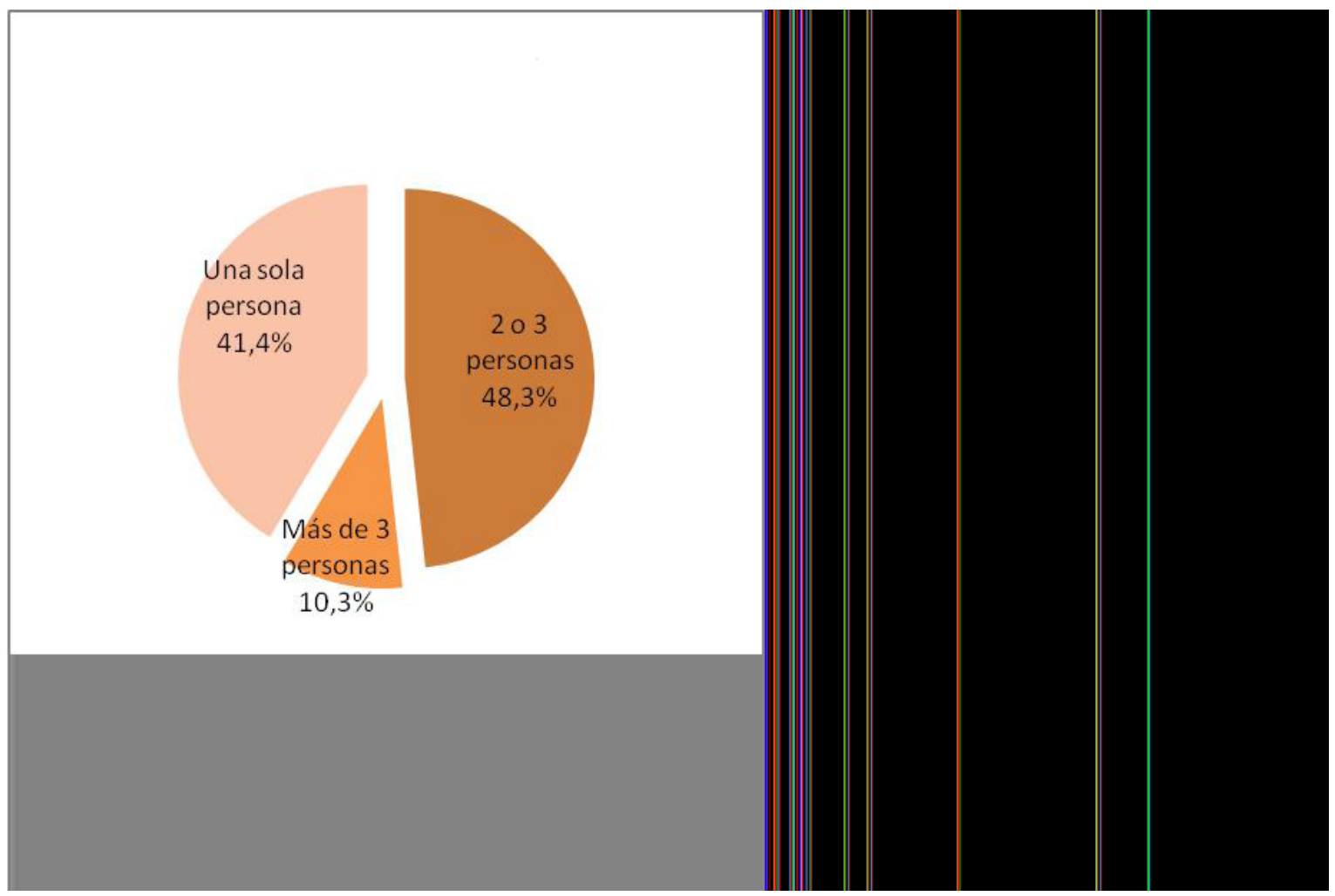

En cuanto al segundo objetivo el $58,2 \%$ de entidades náutico deportivas que sí realizan comunicación, el personal que asume funciones de comunicación también realiza tareas de otros departamentos o secciones, tales como de gestión, administración, contabilidad y atención a los socios, entre otras. En cambio, el 11,4\% consideran que son actividades puntuales que realiza y que puede asumir cualquier persona de la entidad para una acción determinada. Estos datos son muy significativos puesto que las labores de comunicación son desarrolladas por personas, que se integran en otros departamentos realizando otro tipo de funciones.

Gráfico 3. Estructura del departamento de comunicación

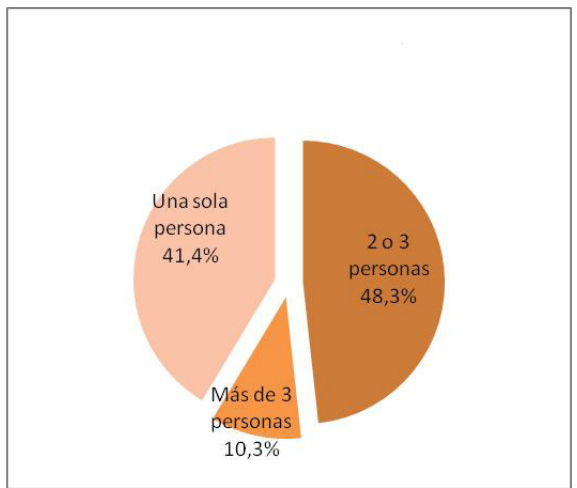

Fuente: Elaboración propia 
El personal de las entidades que sí tienen departamento de comunicación está formado en un $48,3 \%$ por 2 ó 3 personas y en un $41,4 \%$ por una sola persona. Es un resultado importante en cuanto a que este departamento está considerado y valorado para llevar a cabo acciones de comunicación.

Gráfico 4. Formación académica del departamento de comunicación

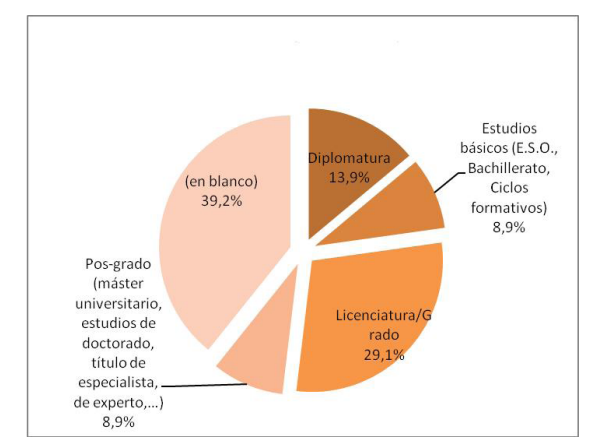

Fuente: Elaboración propia

Un dato reseñable es la formación académica del personal, que asume mayor grado de responsabilidad en el departamento de comunicación. Observamos que la mayoría de clubes no responden, y de los que responden, el 29,1\% afirman que la formación académica es de Licenciatura/Grado y el 13,9\% de Diplomatura. Un dato significativo que demuestra que los departamentos de comunicación de estas entidades están compuestos por personal cualificado y profesional sobre la materia a desempeñar.

\subsection{Las funciones y medios de comunicación del departamento de comunicación}

En cuanto al tercer objetivo (O.3) las entidades deportivas náuticas emplean una serie de funciones para llegar a sus públicos de interés. Estudiaremos qué medios de comunicación son los que más utilizan para comunicarse con sus socios.

Las tareas de comunicación que predominan en las entidades son: mantenimiento y gestión de contenidos de la página web de la entidad (68,35\%), redacción de notas de prensa para medios generalistas sobre la realización de competiciones, actividades y resultados deportivos (60,8\%), redacción de notas de prensa para medios especializados en el sector náutico sobre la realización de competiciones, actividades y resultados deportivos $(59,5 \%)$ y organización y ejecución de eventos deportivos $(59,5 \%)$.

En cambio, otras tareas de comunicación que son desarrolladas en menor medida son: monitorización de interacción con los usuarios en redes sociales, antes, durante y tras la celebración de eventos deportivos, sociales o de otra índole (19\%), análisis del contenido del material publicado en medios impresos $(16,5 \%)$, contratación externa para gestionar las redes sociales y el contenido de la página web $(12,7 \%)$ y la asistencia a ferias para dar a conocer los servicios que ofrece la entidad. 
Estos datos reflejan que siguen una comunicación convencional pero se observa que, poco a poco, se van abriendo a las nuevas tecnologías gestionando el portal web de la entidad.

Gráfico 5. Funciones de comunicación

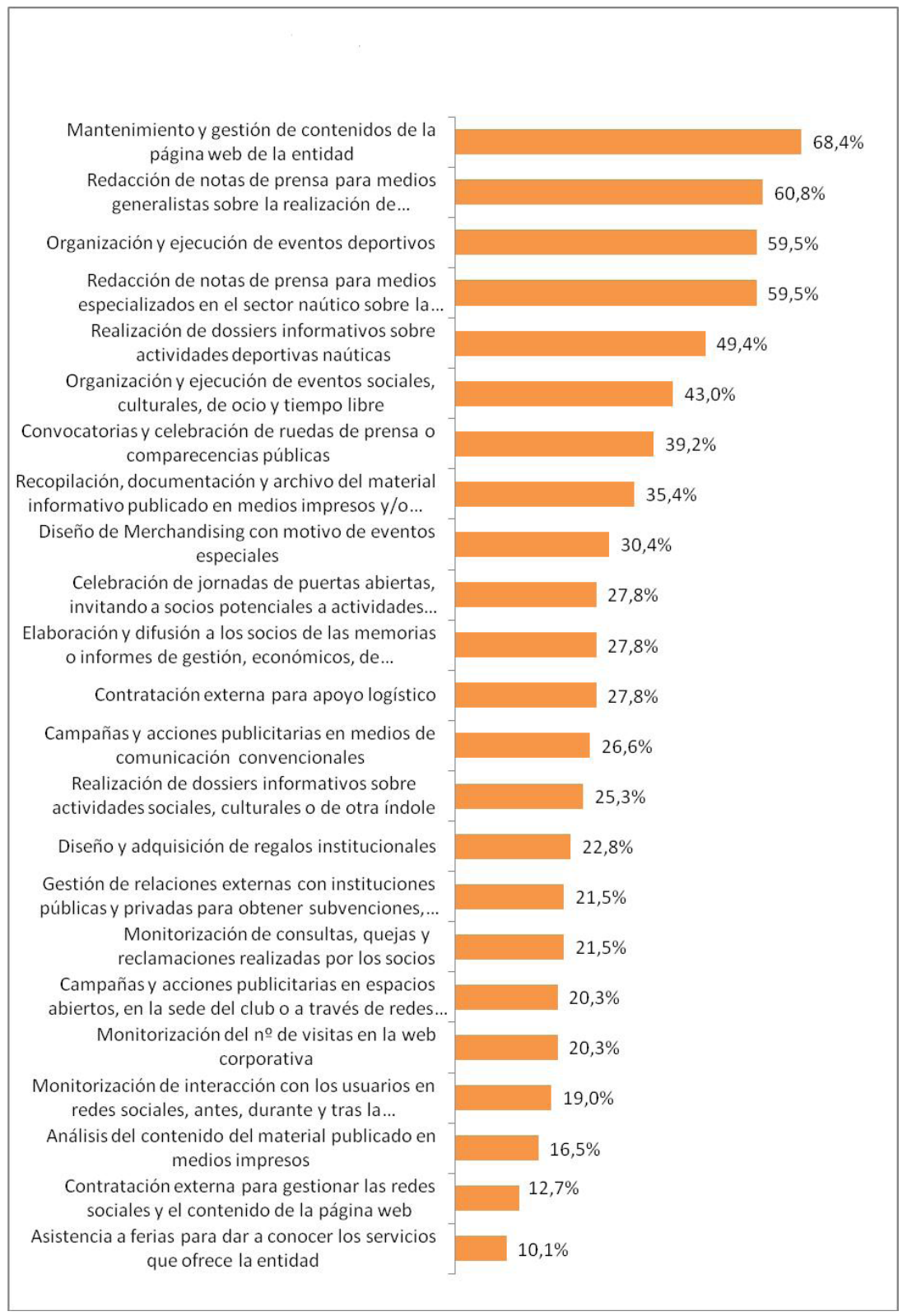

Fuente: Elaboración propia 
Gráfico 6. Medios de comunicación con socios

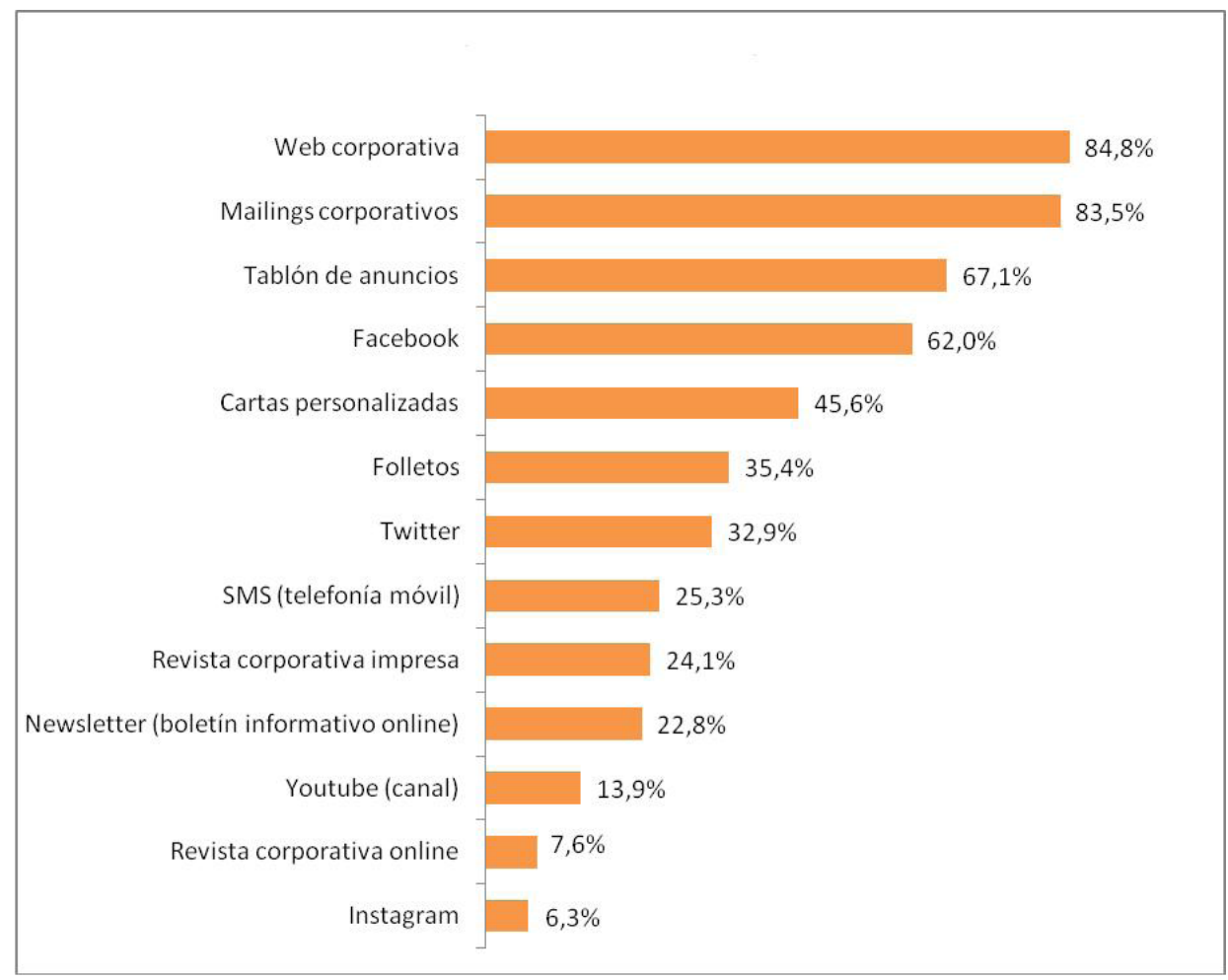

Fuente: Elaboración propia

Como podemos apreciar que los medios de comunicación que se utilizan con mayor frecuencia con los socios son: web corporativa $(84,8 \%)$, mailings corporativos $(83,5 \%)$, tablón de anuncios $(67,1 \%)$ y Facebook (62\%). El resto de medios de comunicación no llegan a ser utilizados por más de la mitad $(50 \%)$ de las entidades. Un resultado favorable para estas entidades que poco a poco se van comprometiendo con las nuevas tecnologías de la comunicación.

\section{Discusión de resultados}

Los resultados alcanzados en relación con el O.1. muestran que estas entidades deportivas náuticas aún les queda camino por recorrer para desarrollar e implantar el departamento de comunicación en su organización. Si comparamos los datos con el I Simposio Nacional de Clubes Náuticos celebrado en el año 2011, este tipo de entidades dan importancia a estos departamentos porque consideran que son esenciales para que este sector evolucione y para que no se quede estancada la idea de que los deportes náuticos son sólo para clases privilegiadas, sino que estas actividades están al alcance de todos.

También vemos conveniente comparar los datos de este gráfico con el II Simposio celebrado en el año 2015, en el que estas entidades toman en cuenta que un departamento de comunicación ayuda a movilizar y dar dinamismo a la entidad gracias a las tecnologías, que hoy en día demandan los socios para acceder a los datos de su club o la compra de servicios tal como existe en otros sectores. De nuevo y después de dos años, en el III Simposio del año 2017 se sigue haciendo hincapié en profesionalizar los departamentos de comunicación. Una tarea importante para los 
clubes náuticos que quieren acercarse a la sociedad del siglo XXI.

En cuanto al personal que desempeña las funciones de comunicación (O.2) se observa que el modelo de comunicación estudiado, el Real Club de Regatas de Alicante, coincide en que el departamento de comunicación de esta entidad deportiva náutica desempeña también funciones administrativas, tales como atención al público en general y a los socios en particular para atender cualquier información que les sea necesaria: cumplimiento de buenas prácticas, gestión de inscripciones a competiciones, gestión de pago de recibos y de venta de ropa, entre otras. Además de desempeñar todas estas competencias se les añade las funciones que le asigne temporalmente la dirección.

El responsable de este departamento es el director de orquesta que coordina a todos los músicos para interpretar una misma pieza musical (Álvarez y Caballero, 1997:85 y Martín, 1998:35). Por lo tanto, este departamento es el encargado de la gestión de comunicación e imagen corporativa de la entidad náutico deportiva (Villafañe, 1999:30-31). Desde esta área se deben coordinar todas las acciones de comunicación para que haya coherencia entre unas y otras. Así mismo deben existir al menos dos áreas: comunicación interna y comunicación externa, que deben de interrelacionarse y llevar a cabo un plan estratégico dirigido por el director de comunicación (Ramírez, 1996:1-6).

En estos departamentos, tal como indicaba Martín (1998:35) se deben integrar por expertos y comunicadores hábiles, que deben estar impregnados de la cultura corporativa de la entidad a la que sirven y que dependen directamente de la alta dirección. De este modo, debe responder a un perfil profesional activo, organizado y estable.

Respecto a las funciones y medios de comunicación que emplean estos departamentos (O.3) se observa que según el modelo investigado, el Real Club de Regatas de Alicante, los resultados coinciden con las funciones del departamento de comunicación de este tipo de entidades deportivas náuticas, siendo éstas: la redacción y envío de convocatorias y notas de prensa; mantenimiento de todas las herramientas de comunicación 2.0, tales como web y redes sociales, entre otras; gestión de protocolo de las competiciones deportivas y eventos/ actos sociales.

Además de todas estas funciones el departamento de comunicación de los clubes náuticos se gestiona: el protocolo del Presidente; relaciones públicas de la entidad; coordinación de eventos; asesorar la imagen corporativa; elaboración de envíos de mailings masivos del club; difusión de eventos y competiciones en los medios de comunicación; gestión de compras e inventario del merchandising; apoyo en la planificación de competiciones y búsqueda de patrocinadores, entre otras. También este tipo de departamentos desempeñan funciones administrativas, tales como atención al público en general y a los socios para cualquier información, gestión de pago de recibos y de venta de ropa. Además de todas las funciones que le asigne temporalmente la dirección.

Los medios de comunicación empleados para comunicarse con sus socios, estas entidades deportivas náuticas sí que han aprovechado las nuevas tecnologías creando sus propias webs corporativas para dar imagen y prestigio a la entidad, pero no han puesto en práctica los blogs ni los boletines oficiales. En cambio, las redes sociales, como por ejemplo Facebook, sí que ha sido un recurso de comunicación utilizado por los clubes náuticos (Olabe, 2009:121-137 y Celaya, 2011:24-27). Son las plataformas para que estas entidades deportivas náuticas difundan 
información y noticias a sus seguidores (Cole, 2016:15) porque les permite elaborar y compartir contenidos (Lobillo y Muñoz, 2016:195-214).

Siguiendo con estos autores, sí que es cierto que los departamentos de comunicación de los clubes náuticos no han aprovechado, suficientemente, las nuevas herramientas de comunicación del medio online porque se echa en falta una mayor participación social online en la vida de los clubes náuticos. No han reconocido que el medio online es una importante herramienta de marketing para ofrecer sus servicios y actividades (Olabe, 2015:83-14). Por lo tanto, a estas entidades deportivas les queda mucho recorrido para que funcionen como entidades 2.0.

Si seguimos comparando estos datos con el II y III Simposio de Clubes Náuticos, observamos que los clubes náuticos son conscientes que tienen que apostar por el marketing online porque es una herramienta que les ayudará a transmitir experiencias a los socios, clientes y consumidores de servicios náuticos. Además, son consecuentes que esta nueva era de la conectividad en el que todos estamos conectados mediante un dispositivo móvil o con una tablet, la oferta y servicios que ofrecen los clubes náuticos deben de ser accesibles a estos medios para llegar a sus socios y al público en general. Gracias a las nuevas tecnologías, este tipo de entidades náutico deportivas serán más dinámicas para que todos sus públicos puedan acceder a los datos de su club o servicios como cualquier otra organización.

\section{Conclusiones}

La investigación desarrollada revela que el sector deportivo náutico está desaprovechando la labor de los departamentos de comunicación. Estos departamentos son importantes para dar a conocer sus servicios a los públicos de interés y fidelizar a los socios que forman parte de este tipo de entidades. Contemplan que estos departamentos son necesarios pero les cuesta incorporarlos, en su día a día, para afrontar los retos del futuro y su supervivencia.

Estas entidades entienden que en este siglo XXI tienen que evolucionar y profesionalizar los departamentos de comunicación para afrontar su supervivencia, pero aún no comprenden que esta área es una pieza clave, que les ayudará a perdurar en el tiempo. Así mismo las entidades que sí hacen comunicación atribuyen a éste departamento, funciones de otras secciones, tales como de gestión, administración, contabilidad, atención al socio, sin permitirles que desarrollen completamente y de manera eficaz las acciones de comunicación del mismo.

El estudio desarrollado nos ha permitido corroborar que los recursos humanos del departamento de comunicación están formados por personas cualificadas y con un alto grado de profesionalización en su área. Los responsables del departamento de comunicación realizan funciones de comunicación y en muchas ocasiones desempeñan labores de otros departamentos.

Los resultados de esta investigación apuntan a que estas entidades no apuestan, de momento, por las nuevas tecnologías pero van poco a poco incorporándose a la era digital. Los clubes náuticos siguen desarrollando por una comunicación convencional, para comunicarse con sus socios, y en menor medida, mediante las webs y redes sociales, están presentes en el mundo online.

A partir de esta investigación podemos afirmar que estas entidades deportivas náuticas sienten la necesidad de comunicar sus servicios y actividades para sobrevivir en este siglo XXI, mediante un departamento de comunicación interno, y por otro, las áreas de comunicación de estas 
entidades no son considerados y valorados puesto que no desempeñan en su totalidad funciones de comunicación sino que realizan funciones de otros departamentos. Es una comunicación corporativa, de imagen de marca, más que orientada a un plan de comunicación para llegar a sus públicos de interés.

Los análisis y resultados obtenidos, sobre el departamento de comunicación en entidades deportivas náuticas son aportaciones significativas que sientan las bases de futuras investigaciones para extrapolarlas a otros ámbitos deportivos. Para ello nos planteamos, como futuras líneas de trabajo, analizar el grado de profesionalización de los departamentos de comunicación de este tipo de entidades, al igual que conocer la formación del personal que dirigen estos departamentos y por último, estudiar los procesos comunicativos y de gestión relacional que desempeñan este tipo de organizaciones con sus stakeholders.

\section{Bibliografía}

Álvarez, T. y Caballero, M. (1997). Vendedores de imagen. Los retos de los nuevos gabinetes de comunicación. Barcelona: Paidós.

Anuario de la Comunicación (2015). El estado de la comunicación en España 2015. Recuperado de: http://www.dircom.org/images/Anuario-Dircom-2015.pdf [Consulta: 20 junio 2018]

Asociación de Clubes Náuticos de la Comunidad Valenciana (ACNCV) (2011). Conclusiones al I Simposio de Clubes Náuticos. Recuperado de: http://www.acncv.org/wp-content/ uploads/2014/07/CONCLUSIONES-SIMPOSIO-NACIONAL-DE-CLUBES-20111.pdf [Consulta: 2 septiembre 2014]

Báez, C. J. (2000). La comunicación efectiva. Santo Domingo: Intec

Billorou, O.P. (1997). Introducción a la Publicidad. Buenos Aires: El Ateneo

Castillo, A., Fernández, M., \& Castillero, E. (2016). Fútbol y redes sociales. Análisis de la gestión de Relaciones Públicas 2.0 por los clubes de fútbol. Estudios sobre el Mensaje Periodístico, 22(1), 239- 254.

Celaya, J. (2011). La empresa en la web 2.0: El impacto de las redes sociales y las nuevas formas de comunicación online. Barcelona: Gestión 2000.

Cole, J. (2016). Examining Facebook Practice: The Case of New Zealand Provincial Rugby. Massey University, Palmerston North, New Zealand

Confederación Española de Asociaciones de Clubes Náuticos (CEACNA) (2011). Importante consenso en el Simposio Nacional de Clubes Náuticos. Recuperado de: http://www.ceacna.com/ noticias2011_1.html [Consulta: 1 junio 2012]

Confederación Española de Asociaciones de Clubes Náuticos (CEACNA) (2015). Conclusiones al II Simposio de Clubes Náuticos. Recuperado de: http://www.simposioclubsnauticos.es/uploaded_files/Document_2190_20151214181055_ es.pdf [Consulta: 15 febrero 2016] 
Comunicación externa (2015). Comunicación global. Disponible en: http://www.cglobal.com.ar/ servicios/20comunicacion-institucional/comunicacion-externa/\%20 [Consulta: 8 abril 2015]

David, M. y Millward, P. (2015) Sport and new Media. En Giulianotti, R. (ed): Routledge Handbook of the Sociology of Sport. London: Routledge

De Asís, A., Gross, D., Lillo., E. y Caro, A. (2002). Manual de ayuda para la gestión de entidades no lucrativas. Madrid: Fundación Luis Vives.

De Moragas, M. (2007, Noviembre). Comunicación y deporte en la era digital. Comunicación presentado en el IX Congreso de la Asociación Española de Investigación Social Aplicada al Deporte (AEISAD), Palmas de Gran Canaria. Resumen recuperado de: http://olympicstudies. uab.es/pdf/wp107_spa.pdf

García, B. (2005). Los altavoces de la actualidad: radiografía de los gabinetes de comunicación. La Coruña: Netbiblo.

Herranz de la Casa, J.M. (2010). La comunicación y la transparencia en las organizaciones no lucrativas (Tesis doctoral no publicada). Universidad Complutense, Madrid.

Lobillo, G. y Muñoz, A. (2016). El papel de las redes sociales en las estrategias de expansión de mercado en los clubes de fútbol españoles. El uso personalizado de Twitter en lengua árabe del Real Madrid CF y FC Barcelona. Trípodos, 38, 195-214.

Llopis- Goig, R. (2016). Deporte, medios de comunicación y sociedad. RICYDE. Revista Internacional de Ciencias del Deporte, XII (44), 86-89.

Martín, F. (1998). Comunicación empresarial e institucional. Madrid: Universitas.

Olabe, F. (2009). La comunicación no convencional en los clubes de fútbol. Pensar la publicidad, 3(1), 121-137. Recuperado de: http://revistas.ucm.es/index.php/PEPU/article/view/ PEPU0909120121A/15348 original/FCB54_Desembre_CAST_baixa.v1325669623.pdf

Olabe, F. (2015). El gabinete de comunicación como impulsor de la gestión comunicativa de los clubes de fútbol. Revista Mediterránea de Comunicación 6. España, pp. 83-104. Disponible en: http//dialnet.uniroja.es/descarga/articulo/4976212.pdf

Paché, G. y Ika, L. (2017). European Professional Football Club Image and Human Capital Management. International Journal of Business Administration and Management Research, 2 (3) JulSept, 30-34

Ramírez, T. (1996). Gabinetes de comunicación: de la seducción por la imagen a la obsesión por "aparecer". Zer, 1, 1-6. Recuperado de: http://www.ehu.eus/zer/hemeroteca/pdfs/zer0108-ramirez.pdf

Rojas, J.L.; Panal, A. (2017). El uso de Instagram en los medios de comunicación deportivos. Análisis comparado de Bleacher Report, L’équipe y Marca. Ámbitos, 38, 1-19. Recuperado de: http:/ /institucional.us.es/ambitos/?p=3123 
Sanahuja, G. (2013). Planificación y estrategia de identidad corporativa en los clubes deportivos españoles. Questiones publicitarias, 1 (18), 126-143. Recuperado de: https://dialnet.unirioja.es/ servlet/articulo?codigo $=4595849$

Segundo Simposio de Clubes Náuticos. Recuperado de: http://www.simposioclubsnauticos.es [Consulta: 15 febrero 2016]

Tercer Simposio de Clubes Náuticos. Recuperado de: http://www.simposioclubsnauticos.es [Consulta: 10 noviembre 2016]

Vázquez, M.A. (2005). Sobre Juego, Deporte y Comunicación, en J. Marín Montín coord., Comunicación y deporte. Nuevas Perspectivas de análisis, (pp. 9-17). Sevilla: Comunicación Social.

Villafañe, J. (1999). La gestión Profesional de la Imagen Corporativa. Madrid: Pirámide.

Xifra, J. (2011). Manual de relaciones públicas e institucionales. Madrid: Tecnos. 
MHCJ no 9 (2) | Año 2018 - Artículo no 18 (133) - Páginas 471 a 490 - mhjournal.org

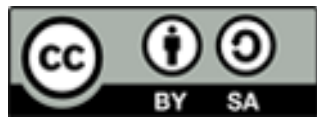

Licencia Creative Commons

Miguel Hernández Communication Journal

mhjournal.org

\section{Cómo citar este texto:}

Paula González Redondo, Concepción Campillo Alhama, Juan Monserrat Gauchi (2018): El Departamento de comunicación de las entidades deportivas náuticas, en Miguel Hernández Communication Journal, n99 (2), pp. 471 a 490. Universidad Miguel Hernández, UMH (Elche-Alicante). DOI: http://dx.doi.org/10.21134/mhcj. v0i9.258 